CUBO A Mathematical Journal

Vol.18, No 01 , (01-14). December 2016

\title{
Uniqueness of meromorphic functions sharing a set in annuli
}

\author{
Renukadevi S. Dyavanal, Ashwini M. Hattikal, Madhura M. Mathai \\ Department of Mathematics, \\ Karnatak University, \\ Dharwad - 580003, India \\ renukadyavanal@gmail.com, ashwinimhmaths@gmail.com, madhuramathai@gmail.com
}

\begin{abstract}
The purpose of this article is to investigate the uniqueness of meromorphic functions sharing a set with counting multiplicity and also with weight 1 in annuli.

\section{RESUMEN}

El propósito de este artículo es investigar la unicidad de funciones meromorfas compartiendo un conjunto contando multiplicidad y también con peso 1 en un anillo.
\end{abstract}

Keywords and Phrases: Annuli, Meromorphic, Sharing Set, Uniqueness.

2010 AMS Mathematics Subject Classification: 30D35, 39A05. 


\section{Introduction and Main Results}

We assume the reader is familiar with standard results and notations of Nevanlinna's theory of meromorphic functions [4],[10],[11]. The purpose of this paper is to study the uniqueness of meromorphic functions in doubly connected domains of complex plane $\mathbb{C}$. By the doubly connected mapping theorem [1], each doubly connected domain is conformally equivalent to the annulus $\{z: r<|z|<R\}, 0 \leq r<R \leq+\infty$. We consider only two cases: $r=0, R=+\infty$ simultaneously and $0<r<R<+\infty$. In the latter case, the homothety $z \longmapsto \frac{z}{\sqrt{r R}}$ reduces the given domain to the annulus $\mathbb{A}=\left\{z: \frac{1}{R_{0}}<|z|<R_{0}\right\}$, where $R_{0}=\sqrt{\frac{R}{r}}$. Thus, in two cases every annulus is invariant with respect to the inversion $z \longmapsto \frac{1}{z}$. Hence, we consider the uniqueness of mermorphic functions in the annulus $\mathbb{A}=\left\{z: \frac{1}{\mathrm{R}_{0}}<|z|<\mathrm{R}_{0}\right\}$, where $1<\mathrm{R}_{0} \leq+\infty$. We denote by $\mathrm{S}$ the subset of distinct elements in $\overline{\mathbb{C}}=\mathbb{C} \cup\{\infty\}$. For a meromorphic function $f$ in $\mathbb{A}$, we define

$$
\begin{aligned}
& E^{\mathbb{A}}(S, f)=\cup_{a} \in S\left\{z \in \mathbb{A}: f_{a}(z)=0, \text { counting multiplicities }\right\}, \\
& \bar{E}^{\mathbb{A}}(S, f)=\cup_{a \in S}\left\{z \in \mathbb{A}: f_{a}(z)=0, \text { ignoring multiplicities }\right\},
\end{aligned}
$$

where $f_{a}(z)=f(z)-a$ if $a \in \mathbb{C}$. We also define

$$
\overline{\mathrm{E}}_{1}^{\mathbb{A}}(\mathrm{S}, \mathrm{f})=\cup_{\mathrm{a} \in \mathrm{S}}\left\{z \in \mathbb{A}: \text { all the simple zeros of } \mathrm{f}_{\mathrm{a}}(z)\right\} \text {. }
$$

For any constant $a$, we say that $f$ and $g$ share a Counting Multiplicity(CM), provided that $f-a$ and $g-a$ have the same zeros with same multiplicities. Similarly, we say that $f$ and $g$ share a Ignoring Multiplicity(IM), provided that $f-a$ and $g-a$ have the same zeros ignoring multiplicities.

In 2009, Cao et al.[3] obtained an analog of Nevanlinna's famous five -value theorem as follows.

Theorem 1.1. Let $\mathrm{f}_{1}$ and $\mathrm{f}_{2}$ be two transcendental or admissible meromorphic functions on the annulus $\mathbb{A}=\left\{z: \frac{1}{\mathrm{R}_{0}}<|z|<\mathrm{R}_{0}\right\}$, where $1<\mathrm{R}_{0} \leq+\infty$. Let $\mathrm{a}_{\mathbf{j}}(\mathbf{j}=1,2,3,4,5)$ be five distinct complex numbers in $\overline{\mathbb{C}}$. If $\mathrm{f}_{1}, \mathrm{f}_{2}$ share $\mathrm{a}_{\mathrm{j}}$ IM for $\mathrm{j}=1,2,3,4,5$, then $\mathrm{f}_{1}(z) \equiv \mathrm{f}_{2}(z)$.

In 2012, Cao and Deng [2] investigated the uniqueness of two meromorphic functions in $\mathbb{A}$ sharing two or three finite sets and obtained the following theorems.

Theorem 1.2. Let $\mathrm{f}$ and $\mathrm{g}$ be two admissible meromorphic functions in the annulus $\mathbb{A}$. Put $\mathrm{S}_{1}=$ $\{0\}, S_{2}=\{\infty\}$, and $S_{3}=\{w: P(w)=0\}$, where

$$
P(w)=a w^{n}+n(n-1) w^{2}+2 n(n-2) b w-(n-1)(n-2) b^{2},
$$

where $\mathrm{n} \geq 5$ is an integer and $\mathrm{a}$ and $\mathrm{b}$ are two non-zero complex numbers satisfying $\mathrm{ab}^{\mathrm{n}-2} \neq 1,2$. If $\overline{\mathrm{E}}\left(\mathrm{S}_{2}, \mathrm{f}\right)=\overline{\mathrm{E}}\left(\mathrm{S}_{2}, \mathrm{~g}\right)$ and $\mathrm{E}\left(\mathrm{S}_{\mathrm{j}}, \mathrm{f}\right)=\mathrm{E}\left(\mathrm{S}_{\mathrm{j}}, \mathrm{g}\right)(\mathrm{j}=1,3)$, then $\mathrm{f} \equiv \mathrm{g}$.

Theorem 1.3. Let $\mathrm{f}$ and $\mathrm{g}$ be two admissible meromorphic functions in the annulus $\mathbb{A}$. Put $\mathrm{S}_{1}=\{\infty\}$ and $\mathrm{S}_{2}=\{w: \mathrm{P}(w)=0\}$, where $\mathrm{P}(\boldsymbol{w})$ is stated as in Theorem 1.2, and $\mathrm{a}$ and $\mathrm{b}$ are two non-zero complex numbers satisfying $\mathrm{ab}^{\mathrm{n}-2} \neq 2, \mathrm{n} \geq 8$ is an integer. If $\overline{\mathrm{E}}\left(\mathrm{S}_{1}, \mathrm{f}\right)=\overline{\mathrm{E}}\left(\mathrm{S}_{1}, \mathrm{~g}\right)$ and $\mathrm{E}\left(\mathrm{S}_{2}, \mathrm{f}\right)=\mathrm{E}\left(\mathrm{S}_{2}, \mathrm{~g}\right)$, then $\mathrm{f} \equiv \mathrm{g}$. 
In 2013, $\mathrm{Xu}$ and $\mathrm{Wu}[8]$ obtained the following Theorems.

Theorem 1.4. Let $\mathrm{f}$ and $\mathrm{g}$ be two admissible meromorphic functions in the annulus $\mathbb{A}$. Let $\mathrm{S}=\left\{w \in \mathbb{A}: \mathrm{P}_{1}(w)=0\right\}$, where $\mathrm{P}_{1}(w)=\frac{(\mathrm{n}-1)(\mathrm{n}-2)}{2} w^{\mathrm{n}}-\mathrm{n}(\mathrm{n}-2) w^{\mathrm{n}-1}+\frac{\mathrm{n}(\mathrm{n}-1)}{2} w^{\mathrm{n}-2}-\mathrm{c}$ and $\mathrm{c}(\neq 0,1)$ is a complex number. If $\mathrm{E}^{\mathbb{A}}(\mathrm{S}, \mathrm{f})=\mathrm{E}^{\mathbb{A}}(\mathrm{S}, \mathrm{g})$ and $\mathrm{n}$ is an integer satisfying $\geq 11$, then $f \equiv g$.

Theorem 1.5. Let $\mathbf{f}$ and $\mathrm{g}$ be two admissible meromorphic functions in the annulus $\mathbb{A}$. If $\mathrm{n}$ is an integer $\geq 7, \mathbb{E}^{\mathbb{A}}(S, f)=\mathbb{E}^{\mathbb{A}}(S, g)$ and $\Theta_{0}(\infty, f)>\frac{3}{4}, \Theta_{0}(\infty, g)>\frac{3}{4}$, where $S$ is defined as in Theorem 1.4, then $\mathrm{f} \equiv \mathrm{g}$.

Theorem 1.6. Let $\mathrm{f}$ and $\mathrm{g}$ be two admissible meromorphic functions in the annulus $\mathbb{A}$. If $\mathrm{E}_{1}^{\mathbb{A}}(\mathrm{S}, \mathrm{f})=\mathrm{E}_{1}^{\mathbb{A}}(\mathrm{S}, \mathrm{g})$ and $\mathrm{n}$ is an integer $\geq 15$, where $\mathrm{S}$ is defined as in Theorem 1.4 , then $\mathrm{f} \equiv \mathrm{g}$.

Theorem 1.7. Let $\mathrm{f}$ and $\mathrm{g}$ be two admissible meromorphic functions in the annulus $\mathbb{A}$. If $\mathrm{n}$ is an integer $\geq 9, \mathrm{E}_{1}^{\mathbb{A}}(S, f)=\mathbb{E}_{1}^{\mathbb{A}}(S, g)$ and $\Theta_{0}(\infty, f)>\frac{5}{6}, \Theta_{0}(\infty, g)>\frac{5}{6}$, where $S$ is defined as in Theorem 1.4, then $\mathrm{f} \equiv \mathrm{g}$.

The main purpose of this paper is to investigate the uniqueness of meromorphic functions sharing a set $S=\{w \in \mathbb{A}: P(w)=0\}$, where $P(w)=w^{\mathfrak{n}}+\alpha w^{n-m}+\beta, \alpha$ and $\beta$ are two non-zero constants different from the sets considered by Cao and Deng [2] and X.Y.Xu and Z.T.Wu [8]. The set considered in this paper is more general, as set varies by varying value of $m$ and constants $\alpha$ and $\beta$ for a fixed $n$, where as the set in [2] and [8] are specific for a fixed $n$.

To prove the main results, we follow the techniques used by X.Y.Xu and Z.T.Wu [8] till the conclusion part and using different technique, conclusion part is effectively proved as the sharing set $S$ is different from the set considered in [8].

Theorem 1.8. Let $\mathrm{f}$ and $\mathrm{g}$ be two admissible meromorphic functions in the annulus $\mathbb{A}$. Let $\mathrm{n}>2 \mathrm{~m}+8$ and $\mathrm{m} \geq 2$ be integers with $\mathrm{n}$ and $\mathrm{m}$ having no common factors. Let $\mathrm{S}=\{\mathrm{w} \in \mathbb{A}$ : $\mathrm{P}(w)=0\}$, where $\mathrm{P}(w)=w^{\mathrm{n}}+\alpha w^{\mathrm{n}-\mathrm{m}}+\beta, \alpha$ and $\beta$ are two non-zero constants such that the algebraic equation $w^{\mathfrak{n}}+\alpha w^{\mathfrak{n}-\mathrm{m}}+\beta=0$ has no multiple roots. If $\mathrm{E}^{\mathbb{A}}(\mathrm{S}, \mathrm{f})=\mathrm{E}^{\mathbb{A}}(\mathrm{S}, \mathrm{g})$, then $\mathrm{f} \equiv \mathrm{g}$.

As inspired by the proof of the Theorem 1.5, Theorem 1.6 and Theorem 1.7, we proved the following Theorems.

Theorem 1.9. Let $\mathrm{f}$ and $\mathrm{g}$ be two admissible meromorphic functions in the annulus $\mathbb{A}$. Let $\mathrm{n}>$ $2 m+5$ and $m \geq 2$ be integers. If $\mathrm{E}^{\mathbb{A}}(S, f)=\mathrm{E}^{\mathbb{A}}(S, g)$ and $\Theta_{0}(\infty, f)>\frac{3}{4}, \Theta_{0}(\infty, g)>\frac{3}{4}$, where $S$ is defined as in Theorem 1.8, then $\mathrm{f} \equiv \mathrm{g}$.

Theorem 1.10. Let $\mathrm{f}$ and $\mathrm{g}$ be two admissible meromorphic functions in the annulus $\mathbb{A}$. Let $\mathrm{n}>2 \mathrm{~m}+12$ and $\mathrm{m} \geq 2$ be integers. If $\mathrm{E}_{1}^{\mathbb{A}}(\mathrm{S}, \mathrm{f})=\mathrm{E}_{1}^{\mathbb{A}}(\mathrm{S}, \mathrm{g})$, where $\mathrm{S}$ is defined as in Theorem 1.8, then $\mathrm{f} \equiv \mathrm{g}$.

Theorem 1.11. Let $\mathrm{f}$ and $\mathrm{g}$ be two admissible meromorphic functions in the annulus $\mathbb{A}$. Let $\mathrm{n}>2 \mathrm{~m}+7$ and $\mathrm{m} \geq 2$ be integers. If $\mathrm{E}_{1}^{\mathbb{A}}(\mathrm{S}, \mathrm{f})=\mathrm{E}_{1}^{\mathbb{A}}(\mathrm{S}, \mathrm{g})$ and $\Theta_{0}(\infty, f)>\frac{5}{6}, \Theta_{0}(\infty, \mathrm{g})>\frac{5}{6}$, where $\mathrm{S}$ is defined as in Theorem 1.8, then $\mathrm{f} \equiv \mathrm{g}$. 


\section{Basic Notations in the Nevanlinna Theory on Annuli}

Let $f$ be a meromorphic function in $\mathbb{C}$. We recall the classical notations of the Nevanlinna theory as follows:

$$
\begin{gathered}
N(R, f)=\int_{0}^{R} \frac{n(t, f)-n(0, f)}{t} d t+n(0, f) \log R \\
m(R, f)=\frac{1}{2 \pi} \int_{0}^{2 \pi} \log ^{+}\left|f\left(R e^{i \theta}\right)\right| d \theta, \quad T(R, f)=m(R, f)+N(R, f)
\end{gathered}
$$

Where $\log ^{+} x=\max \{\log x, 0\}$ and $n(t, f)$ is the counting function of poles of the function $f$ in $\{z:|z| \leq t\}$.The following are the notations and basic results of Nevanlinna theory on annuli $\mathbb{A}=\left\{z: \frac{1}{R_{0}}<|z|<R_{0}\right\}$ for $1<R<R_{0} \leq+\infty$, which can be found in [5] and [6]. Let

$$
N_{1}(R, f)=\int_{\frac{1}{R}}^{1} \frac{n_{1}(t, f)}{t} d t, \quad N_{2}(R, f)=\int_{1}^{R} \frac{n_{2}(t, f)}{t} d t
$$

Where $n_{1}(t, f)$ and $n_{2}(t, f)$ are the counting function of poles of the function $f$ in $\{z: t<|z| \leq 1\}$ and $\{z: 1<|z| \leq \mathrm{t}\}$, respectively. Let

$$
\begin{aligned}
& N_{0}(R, f)=N_{1}(R, f)+N_{2}(R, f), m_{0}(R, f)=m(R, f)+m\left(\frac{1}{R}, f\right) \text { and } \\
& \bar{N}_{0}(r, f)=\bar{N}_{1}(R, f)+\bar{N}_{2}(R, f) \\
&=\int_{\frac{1}{R}}^{1} \frac{\bar{n}_{1}(t, f)}{t} d t+\int_{1}^{R} \frac{\bar{n}_{2}(t, f)}{t} d t
\end{aligned}
$$

Where $\bar{n}_{1}(t, f)$ and $\bar{n}_{2}(t, f)$ are the reduced counting function of poles of the function $f$ in $\{z: t<$ $|z| \leq 1\}$ and $\{z: 1<|z| \leq t\}$, respectively.

Finally, we define the Nevanlinna characteristic of $f$ on the annulus $\mathbb{A}$ by

$$
T_{0}(R, f)=m_{0}(R, f)-2 m(1, f)+N_{0}(R, f)
$$

In addition, we have

$$
\begin{gathered}
\bar{N}_{0}^{(2)}(R, f)=\bar{N}_{0}(R, f)+\bar{N}_{0}^{(2}(R, f), \\
\bar{N}_{0}^{(2}(R, f)=\bar{N}(R, f)-\bar{N}_{0}^{1)}(R, f)
\end{gathered}
$$

where $\bar{N}^{1)}(R, f)$ is the reduced counting function which only counts simple zeros of the function $f$. And the Nevanlinna characteristic of $f$ has the following properties.

(i) $T_{0}(R, f)=T_{0}\left(R, \frac{1}{f}\right)$,

(ii) $\max \left\{T_{0}\left(R, f_{1} \cdot f_{2}\right), T_{0}\left(R, \frac{f_{1}}{f_{2}}\right), T_{0}\left(R, f_{1}+f_{2}\right)\right\} \leq T_{0}\left(R, f_{1}\right)+T_{0}\left(R, f_{2}\right)+O(1)$

By above properties, the first fundamental theorem on the annulus $\mathbb{A}$ is immediately obtained as follows. 
First Fundamental theorem in annuli : Let $f$ be a non-constant meromorphic function on the annulus $\mathbb{A}=\left\{z: \frac{1}{R_{0}}<|z|<R_{0}\right\}$. For $1<R<R_{0} \leq+\infty$, we have

$$
T_{0}\left(R, \frac{1}{f-a}\right)=T_{0}(R, f)+O(1) \quad \text { for every fixed } a \in \mathbb{C} .
$$

Definition 1. For every $\mathrm{a} \in \overline{\mathbb{C}}$, the reduced deficiency is given by

$$
\Theta_{0}(a, f)=1-\limsup _{R \rightarrow \infty} \frac{\bar{N}_{0}\left(R, \frac{1}{f-a}\right)}{T_{0}(R, f)} .
$$

Definition 2. Let $\mathrm{f}$ be a meromorphic function on the annulus $\mathbb{A}=\left\{z: \frac{1}{R_{0}}<|z|<R_{0}\right\}$, where $1<\mathrm{R}_{0} \leq+\infty$. The function $\mathrm{f}$ is called an admissible meromorphic function on the annulus $\mathbb{A}$ provided that

or

$$
\limsup _{R \rightarrow \infty} \frac{T_{0}(R, f)}{\log R}=\infty, \quad 1<R<R_{0}=+\infty,
$$

$$
\limsup _{R \rightarrow R_{0}} \frac{T_{0}(R, f)}{-\log \left(R_{0}-R\right)}=\infty, \quad 1<R<R_{0}<+\infty
$$

respectively.

Khrystiyanyn and Kondratyuk[5] obtained the following lemma on the logarithmic derivative on the annulus $\mathbb{A}$.

Lemma on the logarithmic derivative : Let $\mathrm{f}$ be a non-constant meromorphic function on the annulus $\mathbb{A}=\left\{z: \frac{1}{R_{0}}<|z|<R_{0}\right\}$, where $R_{0} \leq+\infty$ and let $\lambda>0$. Then,

(I) If $R_{0}=+\infty$, then $m_{0}\left(R, \frac{f^{\prime}}{f}\right)=O\left(\log \left(R T_{0}(R, f)\right)\right)$, for $R \in(1,+\infty)$ except for the set $\Delta_{R}$ such that $\int_{\Delta_{R}} R^{\lambda-1} d R<+\infty$;

(II) If $R_{0}<+\infty$, then $m_{0}\left(R, \frac{f^{\prime}}{f}\right)=O\left(\log \left(\frac{T_{0}(R, f)}{R_{0}-R}\right)\right)$, for $R \in\left(1, R_{0}\right)$ except for the set $\Delta_{R}^{\prime}$ such that $\int_{\Delta_{R}^{\prime}} \frac{d R}{\left(R_{0}-R\right)^{\lambda-1}}<+\infty$.

\section{Some Lemmas}

For the proof of our main results, we need the following lemmas.

Lemma 2.1. ([3]) Let $\mathrm{f}$ be a non-constant meromorphic function on the annulus $\mathbb{A}=\left\{z: \frac{1}{\mathrm{R}_{0}}<\right.$ $\left.|z|<\mathrm{R}_{0}\right\}$, where $1<\mathrm{R}_{0} \leq+\infty$. Let $\mathrm{a}_{1}, \mathrm{a}_{2}, \cdots, \mathrm{a}_{\mathrm{q}}$ be $\mathrm{q}$ distinct complex numbers in the extended complex plane $\overline{\mathbb{C}}$. Then,

$$
(q-2) T_{0}(R, f)<\sum_{j=1}^{q} \bar{N}_{0}\left(R, \frac{1}{f-a_{j}}\right)+S(R, f)
$$


(1) If $\mathrm{R}_{0}=+\infty$, then $\mathrm{S}(\mathrm{R}, \mathrm{f})=\mathrm{O}\left(\log \left(\mathrm{R} \mathrm{T}_{0}(\mathrm{R}, \mathrm{f})\right)\right)$, for $\mathrm{R} \in(1,+\infty)$ except for the set $\Delta_{\mathrm{R}}$ such that $\int_{\Delta \mathrm{R}} \mathrm{R}^{\lambda-1} \mathrm{dR}<+\infty$.

(2) If $\mathrm{R}_{0}<+\infty$, then $\mathrm{S}(\mathrm{R}, \mathrm{f})=\mathrm{O}\left(\log \left(\frac{\mathrm{T}_{0}(\mathrm{R}, \mathrm{f})}{\mathrm{R}_{0}-\mathrm{R}}\right)\right)$, for $\mathrm{R} \in\left(1, \mathrm{R}_{0}\right)$ except for the set $\Delta_{\mathrm{R}}^{\prime}$ such that $\int_{\Delta_{\mathrm{R}}^{\prime}} \frac{\mathrm{dR}}{\left(\mathrm{R}_{0}-\mathrm{R}\right)^{\lambda-1}}<+\infty$.

Lemma 2.2. ([2]) Let $\mathrm{f}$ be a non-constant meromorphic function in $\mathbb{A}, \mathrm{Q}_{1}(\mathrm{f})$ and $\mathrm{Q}_{2}(\mathrm{f})$ be two mutually prime polynomials in $\mathrm{f}$ with degree $\mathrm{m}$ and $\mathrm{n}$, respectively. Then,

$$
T_{0}\left(R, \frac{Q_{1}(f)}{Q_{2}(f)}\right)=\max \{m, n\} T_{0}(R, f)+S(R, f),
$$

where $\mathrm{S}(\mathrm{R}, \mathrm{f})$ is defined as in Lemma 2.1 .

Lemma 2.3. ([8]) Let $\mathrm{f}$ be a non-constant meromorphic function in $\mathbb{A}$. Then,

$$
N_{0}\left(R, \frac{1}{f^{\prime}}\right) \leq N_{0}\left(R, \frac{1}{f}\right)+\bar{N}_{0}(R, f)+S(R, f)+O(1)
$$

where $\mathrm{S}(\mathrm{R}, \mathrm{f})$ is defined as in Lemma 2.1 .

Lemma 2.4. ([8]) Let $\mathrm{F}$ and $\mathrm{G}$ be admissible meromorphic functions in $\mathbb{A}$ satisfying $\mathrm{E}^{\mathbb{A}}(\mathrm{F}, 0)=$ $\mathrm{E}^{\mathbb{A}}(\mathrm{G}, 0)$ and $\mathrm{c}_{1}, \mathrm{c}_{2}, \cdots, \mathrm{c}_{\mathrm{q}}$ be $\mathrm{q} \geq 2$ distinct non-zero complex numbers. If

$$
\begin{aligned}
& \limsup _{R \rightarrow \infty, R \in I} \frac{3 \bar{N}_{0}(R, F)+\sum_{j=1}^{q} \bar{N}_{0}^{(2)}\left(R, \frac{1}{F-c_{j}}\right)+\bar{N}_{0}\left(R, \frac{1}{F^{\prime}}\right)}{T_{0}(R, F)}<q \\
& \limsup _{R \rightarrow \infty, R \in I} \frac{3 \bar{N}_{0}(R, G)+\sum_{j=1}^{q} \bar{N}_{0}^{(2)}\left(R, \frac{1}{G-c_{j}}\right)+\bar{N}_{O}\left(R, \frac{1}{G^{\prime}}\right)}{T_{0}(R, G)}<q
\end{aligned}
$$

Where $\overline{\mathrm{N}}_{0}^{(2)}(\mathrm{R}, *)=\overline{\mathrm{N}}_{0}(\mathrm{R}, *)+\overline{\mathrm{N}}_{0}^{(2}(\mathrm{R}, *), \overline{\mathrm{N}}_{0}^{(2}(\mathrm{R}, *)=\overline{\mathrm{N}}_{0}(\mathrm{R}, *)-\overline{\mathrm{N}}_{0}^{1)}(\mathrm{R}, *)$ and I is some set of $\mathbb{R}$ of infinite linear measure, then

$$
F=\frac{a G+b}{c G+d}
$$

where $\mathrm{a}, \mathrm{b}, \mathrm{c}, \mathrm{d} \in \mathbb{C}$ are constants with $\mathrm{ad}-\mathrm{bc} \neq 0$.

Lemma 2.5. ([8]) Let $\mathrm{F}$ and $\mathrm{G}$ be admissible meromorphic functions in $\mathbb{A}$ satisfying $\mathrm{E}_{1}^{\mathbb{A}}(\mathrm{F}, 0)=$ $\mathrm{E}_{1}^{\mathbb{A}}(\mathrm{G}, 0)$ and let $\mathrm{c}_{1}, \mathrm{c}_{2}, \cdots, \mathrm{c}_{\mathrm{q}}$ be $\mathrm{q} \geq 2$ distinct non-zero complex numbers. If

$$
\begin{aligned}
& \limsup _{R \rightarrow \infty, R \in I} \frac{3 \bar{N}_{0}(R, F)+\sum_{j=1}^{q} \bar{N}_{0}^{(2)}\left(R, \frac{1}{F-c_{j}}\right)+\bar{N}_{O}\left(R, \frac{1}{F^{\prime}}\right)+2 \bar{N}_{0}^{(2}\left(R, \frac{1}{F}\right)}{T_{0}(R, F)}<q \\
& \limsup _{R \rightarrow \infty, R \in I} \frac{3 \bar{N}_{0}(R, G)+\sum_{j=1}^{q} \bar{N}_{0}^{(2)}\left(R, \frac{1}{G-c_{j}}\right)+\bar{N}_{O}\left(R, \frac{1}{G^{\prime}}\right)+2 \bar{N}_{0}^{(2)}\left(R, \frac{1}{G}\right)}{T_{0}(R, G)}<q
\end{aligned}
$$


Where $\overline{\mathrm{N}}_{0}^{(2)}(\mathrm{R}, *), \overline{\mathrm{N}}_{0}^{(2}(\mathrm{R}, *)$ and I are stated as in Lemma 2.4, then

$$
F=\frac{a G+b}{c G+d}
$$

where $\mathrm{a}, \mathrm{b}, \mathrm{c}, \mathrm{d} \in \mathbb{C}$ are constants with $\mathrm{ad}-\mathrm{bc} \neq 0$.

Lemma 2.6. [6] Let $\mathrm{f}$ be a non-constant meromophic function on $\mathbb{A}=\{z: 0<|z|<\infty\}$. Let $\left\{\mathrm{a}_{v}\right\}$ be any finite collection of complex numbers possibly including $\infty$. Then,

$$
\sum_{v=1}^{\mathrm{q}} \Theta_{0}\left(a_{v}\right) \leq 2 .
$$

\section{Proof of Theorem 1.1}

If $\mathrm{P}(w)=w^{n}+\alpha w^{n-m}+\beta$, we can get that $\mathrm{P}(0)=\beta=\mathrm{c}_{1}(\neq 0)$

and $P(1)=1+\alpha+\beta=c_{2}(\neq 0)$, and

$$
\begin{gathered}
\mathrm{P}^{\prime}(w)=w^{n-m-1}\left[n w^{m}+\alpha(n-m)\right] \\
P(w)-c_{1}=w^{n-m} Q_{1}(w)
\end{gathered}
$$

Where $\mathrm{Q}_{1}(w)$ is a polynomial of degree $\mathrm{m}$ and $\mathrm{Q}_{1}(0) \neq 0$.

$$
\mathrm{P}(w)-\mathrm{c}_{2}=(w-1) \mathrm{Q}_{2}(w)
$$

Where $\mathrm{Q}_{2}(w)$ is a polynomial of degree $\mathrm{n}-1$ and $\mathrm{Q}_{2}(1) \neq 0$.

From (3.2) and (3.3), we notice that $\mathrm{Q}_{1}(w)$ and $\mathrm{Q}_{2}(w)$ have only simple zeros.

Let $F=P(f)$ and $G=P(g)$. Since $E^{\mathbb{A}}(S, f)=E^{\mathbb{A}}(S, g)$, we get that $F$ and $G$ share the value $0 \mathrm{CM}$. From (3.2) and (3.3), we have

$$
\begin{aligned}
\bar{N}_{0}^{(2)}\left(R, \frac{1}{F-c_{1}}\right) & =\bar{N}_{0}\left(R, \frac{1}{F-c_{1}}\right)+\bar{N}_{0}^{(2}\left(R, \frac{1}{F-c_{1}}\right) \\
& \leq 2 \bar{N}_{0}\left(R, \frac{1}{f}\right)+\sum_{i=1}^{m} \bar{N}_{0}\left(R, \frac{1}{f-a_{i}}\right) \\
& =(m+2) T_{0}(R, f)+S(R)
\end{aligned}
$$

Where $a_{i}(i=1,2, \cdots, m)$ are the zeros of $Q_{1}(w)$ in $\mathbb{A}$ and $S(R)=o\left\{T_{0}(R)\right\}, T_{0}(R)=\max \left\{T_{0}(R, f), T_{0}(R, g)\right\}$. And

$$
\begin{aligned}
\bar{N}_{0}^{(2)}\left(R, \frac{1}{F-c_{2}}\right) & =\bar{N}_{0}\left(R, \frac{1}{F-c_{2}}\right)+\bar{N}_{0}^{(2}\left(R, \frac{1}{F-c_{2}}\right) \\
& \leq \bar{N}_{0}\left(R, \frac{1}{f-1}\right)+\sum_{j=1}^{n-1} \bar{N}_{0}\left(R, \frac{1}{f-b_{j}}\right)
\end{aligned}
$$




$$
=n T_{0}(R, f)+S(R)
$$

Where $b_{j}(j=1,2, \cdots, n-1)$ are the zeros of $Q_{2}(w)$ in $\mathbb{A}$.

From (3.1), we obtain

$$
\begin{aligned}
\bar{N}_{0}\left(R, \frac{1}{F^{\prime}}\right)= & \bar{N}_{0}\left(R, \frac{1}{f^{n-m-1}\left[n f^{m}+\alpha(n-m)\right] f^{\prime}}\right) \\
\leq & \bar{N}_{0}\left(R, \frac{1}{f^{n-m-1}}\right)+\bar{N}_{0}\left(R, \frac{1}{n f^{m}+\alpha(n-m)}\right)+\bar{N}_{0}\left(R, \frac{1}{f^{\prime}}\right) \\
& +S(R)
\end{aligned}
$$

Using Lemma 2.3, (3.6) reduces to

$$
\begin{aligned}
\bar{N}_{0}\left(R, \frac{1}{F^{\prime}}\right) \leq & \bar{N}_{0}\left(R, \frac{1}{f}\right)+\bar{N}_{0}\left(R, \frac{1}{n f^{m}+\alpha(n-m)}\right)+N_{0}\left(R, \frac{1}{f}\right) \\
& +\bar{N}_{0}(R, f)+S(R) \\
\leq & (m+3) T_{0}(R, f)+S(R)
\end{aligned}
$$

From Lemma 2.2, we get

$$
T_{0}(R, F)=n T_{0}(R, f)+S(R)
$$

Using (3.4) - (3.8) in Lemma 2.4 and Since $n>2 m+8$, we get

$$
\begin{gathered}
\limsup _{R \rightarrow \infty, R \in I} \frac{3 \bar{N}_{0}(R, F)+\sum_{j=1}^{2} \bar{N}_{0}^{(2)}\left(R, \frac{1}{F-c_{j}}\right)+\bar{N}_{0}\left(R, \frac{1}{F^{\prime}}\right)}{T_{0}(R, F)} \\
\leq \frac{n+2 m+8}{n}<2
\end{gathered}
$$

Similarly, we obtain

$$
\limsup _{R \rightarrow \infty, R \in I} \frac{3 \bar{N}_{0}(R, G)+\sum_{j=1}^{2} \bar{N}_{0}^{(2)}\left(R, \frac{1}{G-c_{j}}\right)+\bar{N}_{0}\left(R, \frac{1}{G^{\prime}}\right)}{T_{0}(R, G)}<2
$$

Thus, by Lemma 2.4, we have $F \equiv \frac{a G+b}{c G+d}$, where, $a, b, c, d \in \mathbb{C}$ and $a d-b c \neq 0$. Since $E^{\mathbb{A}}(S, f)$ is non-empty and $E^{\mathbb{A}}(S, f)=E^{\mathbb{A}}(S, g)$, we have $b=0, a \neq 0$.

Hence,

$$
F=\frac{a G}{c G+d}=\frac{G}{A G+B}
$$

where $A=\frac{c}{a}$ and $B=\frac{d}{a}$. Now, we consider the following two cases:

Case 1: Suppose $A \neq 0$. From (3.11), we notice that every zero of $P(g)+\frac{B}{A}$ in $\mathbb{A}$ has multiplicity n. 
Next, the case 1 is followed by three following subcases:

Subcase 1: Suppose $\frac{B}{A}=-c_{1}$. From (3.2), we have

$$
P(g)+\frac{B}{A}=g^{n-m}\left(g^{m}+\alpha\right)=g^{n-m}\left(g-a_{1}\right) \cdots\left(g-a_{m}\right)
$$

Where $a_{i}(i=1,2, \cdots, m)$ are non-zero distinct roots of $g$. It follows that every zero of $g$ in $\mathbb{A}$ has multiplicity at least $m$ and every zero of $g-a_{i}$ in $\mathbb{A}$ has multiplicity of at least $n$. Then by Lemma 2.1, we have

$$
\begin{aligned}
(m-1) T_{0}(R, g) \leq & \bar{N}_{0}\left(R, \frac{1}{g}\right)+\bar{N}_{0}\left(R, \frac{1}{g-a_{1}}\right)+\cdots+\bar{N}_{O}\left(R, \frac{1}{g-a_{m}}\right)+S(R, g) \\
\leq & \frac{1}{m} N_{0}\left(R, \frac{1}{g}\right)+\frac{1}{n} N_{0}\left(R, \frac{1}{g-a_{1}}\right)+\cdots+\frac{1}{n} N_{0}\left(R, \frac{1}{g-a_{m}}\right) \\
& +S(R, g) \\
\leq & \left(\frac{1}{m}+\frac{m}{n}\right) T_{0}(R, g)+S(R)
\end{aligned}
$$

Since $m \geq 2$ and $n>2 m+8$, we arrive at a contradiction.

Subcase 2: Suppose $\frac{B}{A}=-c_{2}$. From (3.3), we have

$$
P(g)+\frac{B}{A}=(g-1)\left(g-b_{1}\right)\left(g-b_{2}\right) \cdots\left(g-b_{n-1}\right)
$$

Where $b_{j} \neq 0,1$ are distinct values. For $j=1,2, \cdots, n-1$, consider

$$
\Theta\left(b_{j}, f\right)=1-\limsup _{R \rightarrow \infty} \frac{\bar{N}_{0}\left(R, b_{j}, f\right)}{T_{0}(R, f)}>\frac{1}{2}
$$

We can see that $P(g)+\frac{B}{A}$ has $n$ values satisfying the above inequality. Thus, by Lemma 2.6, we get a contradiction.

Subcase 3: Suppose $\frac{B}{A} \neq-c_{1},-c_{2}$.

By using the same argument as in Subcase 1 or Subcase 2, we get a contradiction.

Case 2: Suppose $A=0$.

If $B \neq 1$, then from (3.11), we have $F=\frac{G}{B}$; that is

$$
P(f)=\frac{1}{B} P(g)
$$

From (3.2) and (3.16), we have

$$
P(f)-\frac{c_{1}}{B}=\frac{1}{B} g^{n-m}\left(g-a_{1}\right)\left(g-a_{2}\right) \cdots\left(g-a_{m}\right)
$$

Since $\frac{c_{1}}{B} \neq c_{1}$, from (3.14), it follows that $P(f)-\frac{c_{1}}{B}$ has at least $n$ distinct zeros $e_{1}, e_{2}, \cdots, e_{n}$. 
Then by applying Lemma 2.1, we have

$$
\begin{aligned}
(n-2) T_{0}(R, f) \leq & \sum_{i=1}^{n} \bar{N}_{0}\left(R, \frac{1}{f-e_{i}}\right)+S(R) \\
\leq & \bar{N}_{0}\left(R, \frac{1}{g}\right)+\bar{N}_{0}\left(R, \frac{1}{g-a_{1}}\right)+\bar{N}_{0}\left(R, \frac{1}{g-a_{2}}\right) \\
& +\cdots+\bar{N}_{0}\left(R, \frac{1}{g-a_{m}}\right)+S(R) \\
\leq & (m+1) T_{0}(R, g)+S(R)
\end{aligned}
$$

By Applying Lemma 2.4 to (3.16) and from (3.18) and since $n>2 m+8$ and $m \geq 2$, we arrive at a contradiction.

Thus, we get $A=0$ and $B=1$, that is $P(f)=P(g)$

$$
\Rightarrow f^{n}+\alpha f^{n-m}=g^{n}+\alpha g^{n-m}
$$

We set $h=\frac{f}{g}$, we substitute $f=h g$ in (3.19), it follows that

$$
g^{n-m}\left[g^{m}\left(h^{n}-1\right)+\alpha\left(h^{n-m}-1\right)\right]=0
$$

If $h$ is a constant. We have from (3.20) that $h^{n}-1=0$ and $h^{n-m}-1=0$, which implies $h=1$ and hence $\mathrm{f} \equiv \mathrm{g}$.

If $h$ is not a constant, then suppose $f^{n} \not \equiv g^{n}$

Now consider,

$$
\begin{gathered}
g^{m}=\frac{-\alpha\left(h^{n-m}-1\right)}{h^{n}-1} \\
g^{m}=\frac{-\alpha\left(h^{n-m-1}+h^{n-m-2}+\cdots+1\right)}{\left(h^{n-1}+h^{n-2}+\cdots+1\right)} \\
g^{m}=\frac{-\alpha\left(h-v^{n-m-1}\right)\left(h-v^{n-m-2}\right) \cdots(h-v)}{\left(h-u^{n-1}\right)\left(h-u^{n-2}\right) \cdots(h-u)}
\end{gathered}
$$

where $v=\exp ((2 \pi i) /(n-m))$ and $u=\exp ((2 \pi i) / n)$. Since $n$ and $m$ have no common factors, we have $v^{j} \neq u^{k}(j=1,2, \cdots, n-m-1 ; k=1,2, \cdots, n-1)$. Suppose that $z_{k}$ is a zero of $h-u^{k}$ of order $p_{k}$. From (3.23), we have $p_{k} \geq m$.

Thus

$$
\bar{N}_{O}\left(R, \frac{1}{h-u^{k}}\right) \leq \frac{1}{m} N_{O}\left(R, \frac{1}{h-u^{k}}\right) \leq \frac{1}{2} T_{0}(R, h)+O(1)
$$


By Lemma 2.1 and from (3.24), we obtain

$$
\begin{aligned}
(n-3) T_{0}(R, h) & <\sum_{k=1}^{n-1} \bar{N}_{0}\left(R, \frac{1}{h-u^{k}}\right)+S(R, h) \\
& \leq \frac{n-1}{2} T_{0}(R, h)+S(R, h)
\end{aligned}
$$

where $S(R, h)$ is defined as in Lemma 2.1.

Since $n>2 m+8$ and $m \geq 2$, we arrive at a contradiction and since $n$ and $m$ have no common factors, we get $f \equiv g$.

This completes the proof of Theorem 1.8.

Proof of Theorem 1.9:

Since $\Theta_{0}(\infty, f)>\frac{3}{4}$ and $\Theta_{0}(\infty, g)>\frac{3}{4}$

It follows that

$$
\limsup _{R \rightarrow \infty} \frac{\bar{N}_{0}(R, f)}{T_{0}(R, f)}<\frac{1}{4}, \limsup _{R \rightarrow \infty} \frac{\bar{N}_{0}(R, g)}{T_{0}(R, g)}<\frac{1}{4}
$$

By applying (3.25), from Lemma 2.4 and since $n>2 m+5$, we deduce

$$
\begin{aligned}
& \limsup _{R \rightarrow \infty, R \in I} \frac{3 \bar{N}_{0}(R, F)+\sum_{j=1}^{2} \bar{N}_{0}^{(2)}\left(R, \frac{1}{F-c_{j}}\right)+\bar{N}_{0}\left(R, \frac{1}{F^{\prime}}\right)}{T_{0}(R, F)} \\
& <\frac{4}{n} \limsup _{R \rightarrow \infty, R \in I} \frac{\bar{N}_{0}(R, f)}{T_{0}(R, f)}+\limsup _{R \rightarrow \infty, R \in I} \frac{(n+2 m+4) T_{0}(R, f)}{n T_{0}(R, f)}<2
\end{aligned}
$$

Similarly, we get

$$
\limsup _{R \rightarrow \infty, R \in I} \frac{3 \bar{N}_{0}(R, G)+\sum_{j=1}^{2} \bar{N}_{0}^{(2)}\left(R, \frac{1}{G-c_{j}}\right)+\bar{N}_{O}\left(R, \frac{1}{G^{\prime}}\right)}{T_{0}(R, G)}<2
$$

Then, from Lemma 2.4, we have $F=\frac{a G+b}{c G+d}$, where $a, b, c, d \in \mathbb{C}$ and $a d-b c \neq 0$. Thus, by using the same argument as that in Theorem 1.8, we can prove the conclusion of Theorem 1.9.

\section{Proof of Theorem 1.10 :}

Since $E_{1}^{\mathbb{A}}(S, f)=E_{1}^{\mathbb{A}}(S, g)$, we have $E_{1}^{\mathbb{A}}(F, 0)=E_{1}^{\mathbb{A}}(G, 0)$

From (3.1)-(3.3), we can get

$$
\bar{N}_{0}^{(2}\left(R, \frac{1}{F}\right)=\sum_{i=1}^{n} \bar{N}_{0}\left(R, \frac{1}{f-d_{i}}\right) \leq \bar{N}_{0}\left(R, \frac{1}{f^{\prime}}\right)
$$


Where $d_{i}(i=1,2, \cdots, n)$ are the distinct zeros of $P(w)$. And from (3.6), (3.29) and by Lemma 2.3, we have

$$
\begin{aligned}
\bar{N}_{0}\left(R, \frac{1}{F^{\prime}}\right)+2 \bar{N}_{0}^{(2}\left(R, \frac{1}{F}\right) \leq & \bar{N}_{0}\left(R, \frac{1}{f}\right)+\bar{N}_{0}\left(R, \frac{1}{n f^{m}+\alpha(n-m)}\right) \\
& +3 N_{0}\left(R, \frac{1}{f}\right)+3 \bar{N}_{0}(R, f)+S(R) \\
\leq & (4+m) T_{0}(R, f)+3 \bar{N}_{0}(R, f)+S(R)
\end{aligned}
$$

Since $n>2 m+12, m \geq 2, T_{0}(R, F)=n T_{0}(R, f)+S(R)$ and using equations (3.4), (3.5) and (3.30), we deduce

$$
\begin{gathered}
\limsup _{R \rightarrow \infty, R \in I} \frac{3 \bar{N}_{0}(R, F)+\sum_{j=1}^{2} \bar{N}_{0}^{(2)}\left(R, \frac{1}{F-c_{j}}\right)+\bar{N}_{0}\left(R, \frac{1}{F^{\prime}}\right)+2 \bar{N}_{0}^{(2}\left(R, \frac{1}{F}\right)}{T_{0}(R, F)} \\
\leq \frac{n+2 m+12}{n}<2
\end{gathered}
$$

Similarly, we get

$$
\limsup _{R \rightarrow \infty, R \in I} \frac{3 \bar{N}_{0}(R, G)+\sum_{j=1}^{2} \bar{N}_{0}^{(2)}\left(R, \frac{1}{G-c_{j}}\right)+\bar{N}_{0}\left(R, \frac{1}{G^{\prime}}\right)+2 \bar{N}_{0}^{(2}\left(R, \frac{1}{G}\right)}{T_{0}(R, G)}<2
$$

Thus, from Lemma 2.5, we have $F=\frac{a G+b}{c G+d}$, where $a, b, c, d \in \mathbb{C}$ and $a d-b c \neq 0$. Hence, by using the same argument as that in Theorem 1.8, we can prove the conclusion of Theorem 1.10.

\section{Proof of Theorem 1.11 :}

Since $\Theta_{0}(\infty, f)>\frac{5}{6}$ and $\Theta_{0}(\infty, g)>\frac{5}{6}$,

It follows that

$$
\limsup _{R \rightarrow \infty} \frac{\bar{N}_{0}(R, f)}{T_{0}(R, f)}<\frac{1}{6}, \limsup _{R \rightarrow \infty} \frac{\bar{N}_{0}(R, g)}{T_{0}(R, g)}<\frac{1}{6}
$$

By Lemma 2.5, (3.31)- (3.33) and since $n>2 m+7$, we deduce

$$
\limsup _{R \rightarrow \infty, R \in I} \frac{3 \bar{N}_{0}(R, F)+\sum_{j=1}^{2} \bar{N}_{0}^{(2)}\left(R, \frac{1}{F-c_{j}}\right)-\bar{N}_{0}\left(R, \frac{1}{F^{\prime}}\right)+2 \bar{N}_{0}^{(2}\left(R, \frac{1}{F}\right)}{T_{0}(R, F)}
$$




$$
<\frac{6}{n} \limsup _{R \rightarrow \infty, R \in I} \frac{\bar{N}_{0}(R, f)}{T_{0}(R, f)}+\limsup _{R \rightarrow \infty, R \in I} \frac{(n+2 m+6) T_{0}(R, f)}{n T_{0}(R, f)}<2
$$

Similarly, we get

$$
\limsup _{R \rightarrow \infty, R \in I} \frac{3 \bar{N}_{0}(R, G)+\sum_{j=1}^{2} \bar{N}_{0}^{(2)}\left(R, \frac{1}{G-c_{j}}\right)+\bar{N}_{0}\left(R, \frac{1}{G^{\prime}}\right)+2 \bar{N}_{0}^{(2}\left(R, \frac{1}{G}\right)}{T_{0}(R, G)}<2
$$

Then, from Lemma 2.5, we have $F=\frac{a G+b}{c G+d}$, where $a, b, c, d \in \mathbb{C}$ and $a d-b c \neq 0$. Thus, by using the same argument as that in Theorem 1.8, we can prove the conclusion of Theorem 1.11.

Remark: The method used in this paper to prove the conclusion part of main results can be applied to the sets of zeros of polynomials containing only three terms including constant term, but not for more than three terms.

Acknowledgements: The authors are grateful to the referee for his/her keen observations, comments and valuable suggestions towards the improvement of the present paper. First author is supported by UGC SAP DRSIII with Ref. No. F.510/3/DRS-III/2016(SAP-I) Dated: 29th Feb. 2016. Second author and third author were supported by UGC's Research Fellowship in Science for meritorious Students, UGC, New Delhi. Ref. No.F.7101/2007(BSR) and Ref. No. KU/Sch/UGC-UPE/2014-15/894.

\section{References}

[1] S.Axler, Harmonic functions from a complex analysis view point, Amer. Math. Monthly 93 (1986), 246-258.

[2] T.B.Cao, Z.S.Deng, On the uniqueness of meromorphic functions that share three or two finite sets on annuli, Proceedings of the Indian Academy of Mathematical Sciences, 122(2)(2012), 203-220.

[3] T.B.Cao, H.X.Yi, H.Y.Xu, On the multiple values and uniqueness of meromorphic functions on annuli, Comp. Math. Appl. 58(7)(2009), 1457-1465.

[4] W.K.Hayman, Meromorphic functions, Claredon Press, Oxford, 1964.

[5] A.YA.Khrystiyanyn, A.A.Kondratyuk, On the Nevanlinna theory for meromorphic functions on annuli-I, Mathematychin Studii, 23(2005), 19-30.

[6] A.YA.Khrystiyanyn, A.A.Kondratyuk, On the Nevanlinna theory for meromorphic functions on annuli-II, Mathematychin Studii, 24(2005), 57-68. 
[7] W.C.Lin, H.X.Yi, Uniqueness theorems for meromorphic functions that share three sets, Complex Variables, 48(4)(2003), 315-327.

[8] H.Y.Xu, Z.J.Wu, The shared set and uniqueness of meromorphic functions on annuli, Abst. Appl. Anal. 2013 (2013), 10 pages(Article ID 758318).

[9] C.C.Yang, X.H.Hua, Uniqueness and value sharing of meromorphic functions, Ann. Acad. Sci. Fenn. Math. 22(1997), 395-406.

[10] C.C.Yang, H.X.Yi, Uniqueness Theory of Meromorphic Functions, Kluwer Academic Publishers, Dordrecht,2003; Chinese original: Science Press, Beijing, 1995.

[11] L.Yang, Value Distribution Theory, Springer-Verlag Berlin, 1993. 